\title{
MASS TRANSFER ON MULTI-STRINGS OF TOUCHING SPHERES
}

\author{
SUSUMU FUKUSHIMA, KATSUHIKo KUSAKA \\ AND SADAMITSU ISHII \\ Department of Chemical Engineering, \\ Kansai University, Suita 564
}

\begin{abstract}
Interfacial areas and liquid-phase volumetric mass-transfer coefficients were measured from chemical absorption of oxygen into sulfite solution and from physical absorption of carbon dioxide into water, respectively, in the cases of one, two, three and seven strings of touching spheres, and in irrigated columns packed with spheres, Raschig rings and Berl saddles. The columns having seven strings of touching spheres used both open and closed configurations of spheres.

When the number of spheres per string is larger than 10 and the number of strings $m$ is 3 and 7 , the empirical equation of interfacial area $a_{t}$ for sphere diameter $d_{p}$ is given in terms of liquid Reynolds number $\operatorname{Re}_{t}\left(=4 \rho_{l} Q_{t} / m \pi d_{p \ell_{l}}\right)$ and the number of contact points of spheres per sphere $q$ as follows:

$$
a_{t} d_{p}=4.8 q^{-1.1} R e_{t}^{\left\{0.27 q^{0.25}\right\}}
$$
\end{abstract}

The equation obtained by replacing $a_{t}$ by $a_{p} /(1-\varepsilon)$ and $R e_{t}$ by $R e_{p}$ in the above equation is applicable to illustrate the data in irrigated columns packed with spheres, $(1-\varepsilon)$ in solid fraction, with an accuracy of $\pm 20 \%$. Suppose $q=4.2$ for Raschig rings and $q=2.8$ for Berl saddles, the predicted values agree with the experimental data.

The Sherwood numbers are presented by the same relation for the cases of one, two, three and seven strings, where the number of touching spheres is larger than 10, as follows:

$$
S h_{t}=k_{l t}^{*} d_{p} / D_{A}=7.1 R e_{i}^{1 / 3} S c^{1 / 2}
$$

The equation obtained by substituting $k_{l t}^{*}$ by $k_{l p}^{*}, 7.1$ by $27 / \phi(1-\varepsilon)$ and $R e_{t}$ by $R e_{p}$ represents the data for columns with a packing of surface shape factor $\phi$.

\section{Introduction}

In gas absorption, packed columns with countercurrent flow are generally operated at gas and liquid flow rates near flooding point. Packed columns with cocurrent downward flow have recently come into use in chemical absorption, because this type of operation has no flooding limitation.

Under low flow rates of gas and liquid, the liquid irrigates the packing and the contact points of the packing. Thus, each packing unit is completely surrounded by the liquid. In this flow condition, the prediction of mass transfer from gas to liquid is of great importance for a fixed bed of catalyst particles with two-phase flow.

When there are no available data for physical and chemical properties such as solubility, diffusivity and chemical rate equation, it is desirable to find a simple laboratory apparatus capable of predicting gas absorption rate in a packed column.

As for a laboratory apparatus applicable to the design and operation of packed columns for gas absorption, a string of disks and spheres is reported

Received March 3, 1977. Correspondence concerning this article should be addressed to $\mathrm{S}$. Fukushima. by Stephens and Morris ${ }^{11}$, and Lynn et $a l^{8)}$, respectively. Davidson et $a l^{3}{ }^{3}$ discussed gas-absorption into liquid film on a string of touching spheres. In comparison with a string of these spheres and columns loaded with spheres, there is a remarkable difference in the number of contact points of spheres per sphere. It is expected that, as the number of these points in multi-strings of touching spheres increases, the static liquid hold-up per sphere increases and the interfacial area per sphere decreases.

The present work concerns the interfacial areas and mass-transfer coefficients in one, two, three and seven strings of touching spheres and in columns packed with spheres, Raschig rings and Berl saddles. The areas and liquid-phase mass-transfer coefficients were measured from oxygen absorption into sulfite solution with cobaltous chloride and physical absorption into water, respectively. The correlations of the multi-strings and the irrigated columns packed with various packings were studied.

\section{Experimental}

\section{1 Multi-strings of touching spheres}

The strings of touching spheres used are shown in 


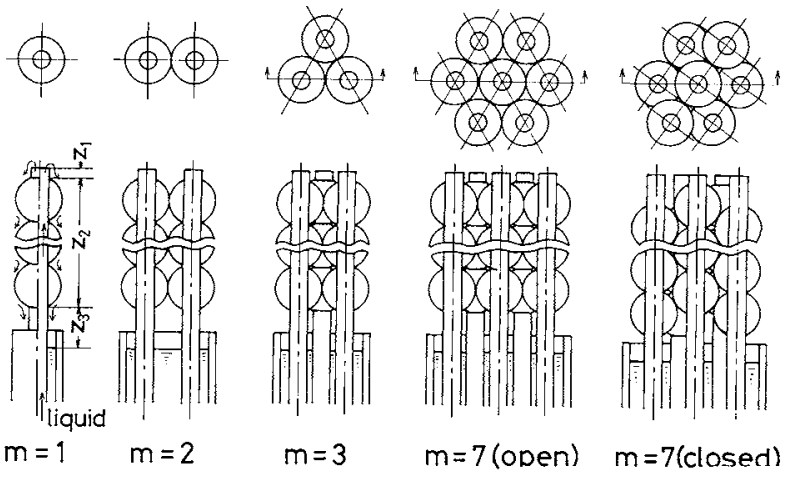

Fig. 1 Strings of touching spheres

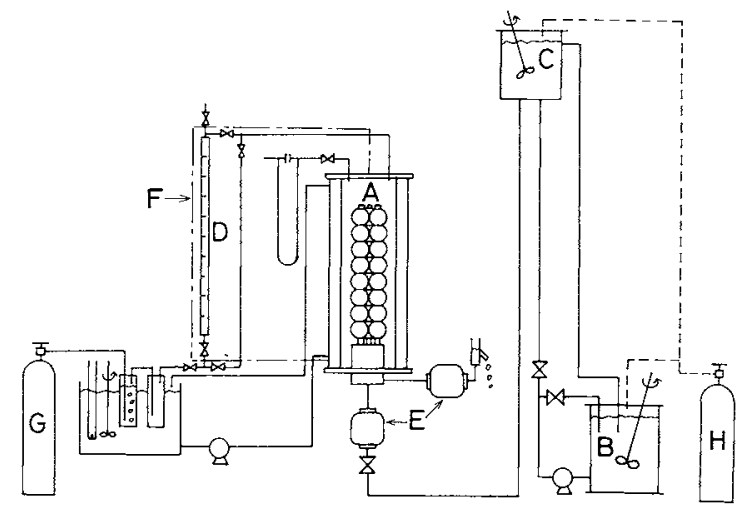

A. strings of touching spheres, B. agitated tank, C. head tank, D. soap film meter, E. electromagnetic flowmeter, F. thermostatic chamber, G. oxygen gas cylinder, H. nitrogen gas cylinder

Fig. 2 Flow diagram for chemical absorption on strings of touching spheres

Fig. 1. The diameters of the stainless steel spheres, $d_{p}$, are 2.54 and $3.81 \mathrm{~cm}$, and the holes made at the center of the spheres were 0.95 and $1.6 \mathrm{~cm}$ in diameter. Ten and twenty spheres were connected through the stainlesss steel tube in the holes. The longitudinal distance for a sphere was thus $0.92 d_{p}$. To make a uniform liquid flow on the spheres, the surfaces of these spheres and the outer surface of the tubes at the top and bottom of the strings were scratched with sandpaper. The inside of the tube at the top of the strings, further, was shaped like a knife edge. A string of spheres, standing vertically on a disk, was touched with other strings of spheres. In the case of seven strings, the spheres were arranged in both open and closed configurations.

The total height of the strings of touching spheres is given by

$$
z_{t}=z_{1}+z_{2}+z_{3}
$$

where $z_{1}$ and $z_{3}$ are the tube length at the top and bottom of the strings, respectively, and $z_{2}$ is the height of touching spheres equivalent to the wetted-wall column. The height of $z_{2}$ consists of the interface on the spheres applicable to the Davidson equation ${ }^{3)}$ of the liquid film and the interface on the static liquid holdup at the contact points. The boundary of pictures taken by a Nikon F camera with $135 \mathrm{~mm}$ lens as described in Appendix 1. Thus, the equation for strings of $N$ spheres is written as

$$
z_{2}=1.45 d_{p}+0.69(N-2) d_{p}+0.20(N-1) d_{p}
$$

where the first, second and third terms are the height of the spheres at the top and bottom, that of other spheres and that at the contact points, respectively.

Oxygen absorption into $0.5 \mathrm{M}$ sulfite solution with $10^{-4}$ to $8 \times 10^{-4} \mathrm{M}$ cobaltous chloride at $30^{\circ} \mathrm{C}$ and $\mathrm{pH} 8$ was performed to determine the interfacial area as described in detail previously ${ }^{6}$.

Figure 2 shows the flow diagram for chemical absorption. The sulfite solution, agitated in the tank sealed by nitrogen gas, flows through the tubes of touching spheres through the head tank, the Hokushin electomagnetic flowmeter and the bottom tank placed below the strings. It trickles on the touching spheres. The reacted solution flows out through the flowmeter and the device to maintain a constant level of liquid in the reservoir below the strings. The strings of touching spheres were placed in the glass cylinder with jacket kept at $30 \pm 0.3^{\circ} \mathrm{C}$. Data on saturated oxygen concentration, diffusivity of oxygen and rate equation of chemical reaction are available in the literature ${ }^{5}$.

Physical absorption of pure carbon dioxide, ethylene and propylene gas into water was performed to get liquid-phase volumetric mass-transfer coefficients. These are obtained from the equation

$$
k_{i t}^{*} a_{t}=\frac{G_{l}}{\rho_{l} z_{t}} \ln \frac{C_{A i}-C_{A o}}{C_{A i}-C_{A z}}
$$

\section{2 Packed column}

The columns used were 11.4 and $20 \mathrm{~cm}$ in diameter, and 90 and $120 \mathrm{~cm}$ in height. The apparatus and the determination of interfacial area by oxygen absorption were described in detail previously ${ }^{6}$. To determine the liquid-phase volumetric mass-transfer coefficient, absorption of carbon dioxide in nitrogen gas into water was performed at $30^{\circ} \mathrm{C}$. The absorption rate was determined by analysis of the solution at the outlet with back titration of $1 / 10-\mathrm{N} \mathrm{NaOH}$ solution.

\section{Results and Discussion}

\section{1 Interfacial area}

1) Multi-string of touching spheres Figure 3 shows the dependence of interfacial area $a_{t}$, on liquid Reynolds number $R e_{t}$, based on the equator of spheres in one, three and seven strings of touching spheres. The dependence varies with the number of strings $m$ and, where $m=7$, in the sphere arrangement. 
Table 1 The values of $q_{\max }, q, K$ and $k$ in strings of touching spheres

\begin{tabular}{ccccc}
$m$ & $q_{\max }$ & $q$ & $K$ & $k$ \\
-1 & 2 & 1.8 & 0.69 & 0.43 \\
2 & 3 & 2.7 & & \\
3 & 4 & 3.8 & 1.1 & 0.38 \\
7 (open) & 8 & 5.5 & 0.76 & 0.40 \\
7 (closed) & 12 & 7.7 & 0.48 & 0.45 \\
\hline
\end{tabular}

For three strings, $a_{t}$ is independent of $N$, where $N$ is larger than 10 . This indicates that oxygen absorption on the tube at the top and bottom is negligible in comparison with that on touching spheres in the string, where $N$ is larger than 10 . Thus, the relationship between $a_{t}$ and $R e_{t}$, obtained by the least square method, gives the following equation.

$$
a_{t} d_{p}=K \operatorname{Re}_{t}^{k}
$$

The values of $K$ and $k$ for respective strings are shown in Table 1.

Let's illustrate, for example, the number of contact points per sphere, $q_{c}$ for the sphere at both top and bottom is 3 , because it is 2 in horizontal and 1 in vertical direction. The value for other spheres is 4 , for it is 2 in both directions. Thus, the value of maximum contact points per sphere $q_{\max }$ is 4 , and that of mean contact points $q$ is 3.8 for $N=10$. The values of $q$ and $q_{\text {max }}$ are shown in Table 1 .

The relationships between $q$ and $K$, and $q$ and $k$ in Fig. 4 give the following equations for three and seven strings.

$$
K=4.8 q^{-1.1}
$$

and

$$
k=0.27 q^{0.25}
$$

Substitution of Eqs. (5) and (6) into Eq. (4) gives

$$
a_{t} d_{p}=4.8 q^{-1.1} \operatorname{Re}_{t}^{\left(0.27 q^{0.25)}\right.}
$$

The solid lines in Fig. 3 are the predictions given by Eq. (7) for three strings and for the two types of seven strings. Equation (7) is not capable of illustrating the data for one string.

2) Packed column In a column packed with spheres, the value of $q_{\max }$ is 12 , but that of $q_{c}$ is 9 for the sphere at the column wall. Where the ratio of sphere diameter to column diameter $d_{p} / d_{T}$ is smaller than 0.2 , the value of $q$ approaches 12 . Substitution of this value into Eqs. (5) and (6) gives $K=0.31$ and $k=0.5$. The empirical equation of interfacial area $a_{p}$ for a column packed with spheres is thus obtained by replacing $a_{t}$ by $a_{p} /(1-\varepsilon)$ and $R e_{t}$ by $R e_{p}$ in Eq. (7) as follows:

$$
\frac{a_{p} d_{p}}{(1-\varepsilon)}=4.8 q^{-1.1} \operatorname{Re}_{p t}^{\left(0.27 q^{0.25)}\right.}
$$

where $\varepsilon$ is the void fraction. Figure 5 shows that the predection agrees with the data with an accuracy of $\pm 20 \%$. Equation ( 8 ) is also written as

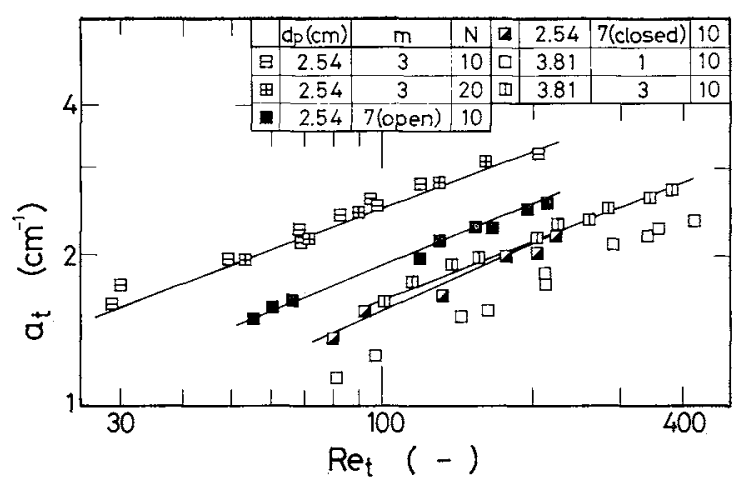

Fig. 3 Dependence of interfacial area on liquid Reynolds number in strings of touching spheres

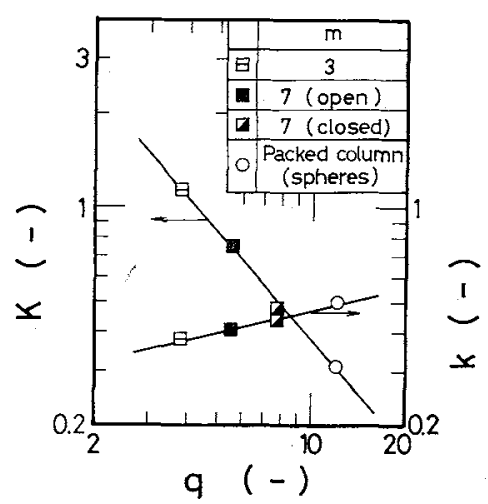

Fig. 4 Relationships between $q$ and $K$, and $q$ and $k$

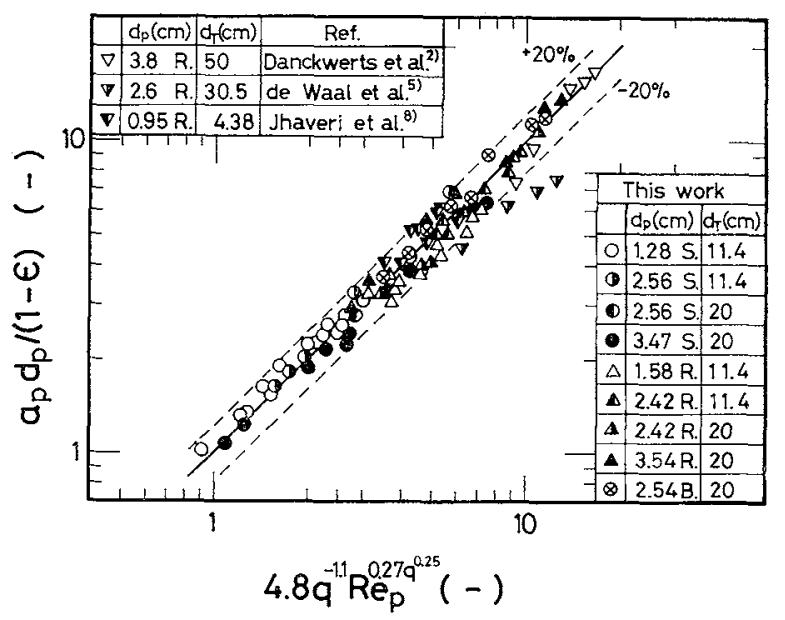

Fig. 5 Correlation of Eq. (8) with the data on columns packed with spheres, Raschig rings and Berl saddles

$$
\frac{a_{p} d_{p}}{(1-\varepsilon)}=0.31 R e_{p}^{0.5}
$$

Assuming Eq. (8) applies to other types of packing, the value of $q$ becomes 4.2 for Raschig rings and 2.8 for Berl saddles. The values of $q, K$ and $k$ for packed columns are shown in Table 2. For Raschig rings, Eq. (8) represents the data presented in this work and those given by Thaveri and Sharma ${ }^{83}$, Danckwerts and Rizvi ${ }^{2)}$, and de Waal and Beek ${ }^{5}$ ? 
Table 2 The values of $K, k, q$ and $\phi$ in packed columns

\begin{tabular}{lllrl} 
& \multicolumn{1}{c}{$K$} & $k$ & $q$ & $\phi$ \\
\hline spheres & 0.31 & 0.50 & 12 & 3.14 \\
Raschig rings & 0.98 & 0.38 & $4.2^{*}$ & 6.15 \\
Berl saddles & 1.6 & 0.35 & $2.8^{*}$ & 4.95 \\
* calculated values from $K$ and $k$ & & \\
\hline
\end{tabular}

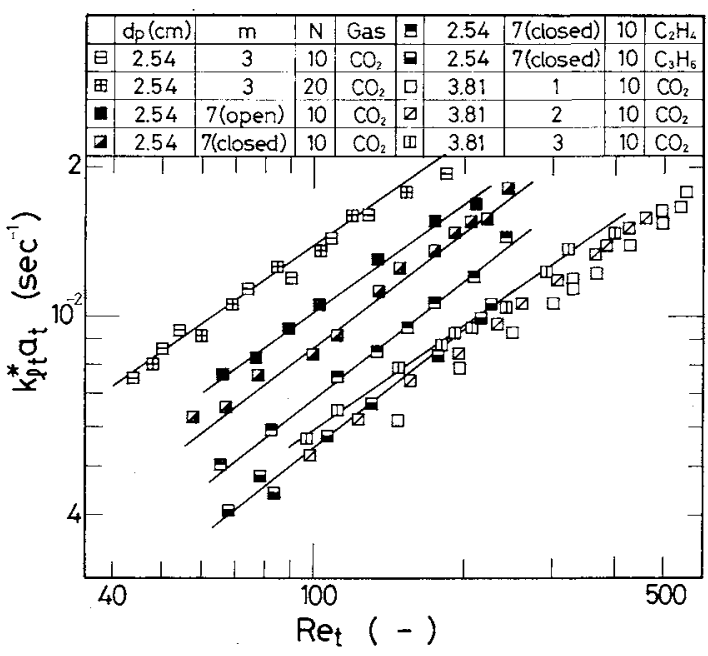

Fig. 6 Dependence of liquid-phase volumetric mass-transfer coefficient on liquid Reynolds number in strings of touching spheres

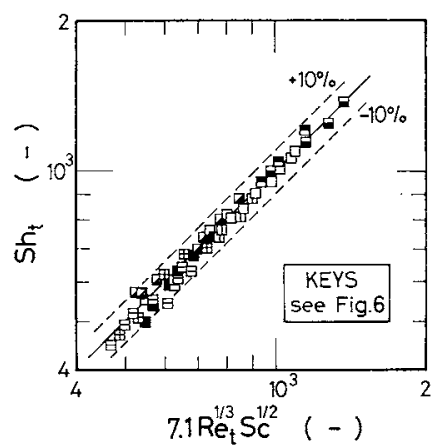

Fig. 7 Correlation of Eq. (10) with the data on strings of touching spheres

with an accuracy of $\pm 20 \%$, as shown in Fig. 5 .

\section{2 Liquid-phase mass-transfer coefficient}

1) Multi-strings of touching spheres Figure 6 shows the dependence of liquid-phase volumetric masstransfer coefficient $k_{l t}^{*} a_{t}$ on $R e_{t}$ for one, two, three and two types of seven strings. For three strings, the coefficient is independent of $N$, where $N$ is larger than 10 .

The data on liquid-phase mass-transfer coefficient $k_{l t}^{*}$ given by dividing $k_{l t}^{*} a_{t}$ by $a_{t}$ are independent of $N, m$ and the type of sphere arrangement, and the coefficient is proportional to $1 / 3$ power of $R e_{t}$. The correlation of the data on gas absorption of carbon dioxide, ethylene and propylene shows that $k_{l t}^{*}$ is proportional to a half power of diffusivity $D_{A}$. Rearrangement of the data gives

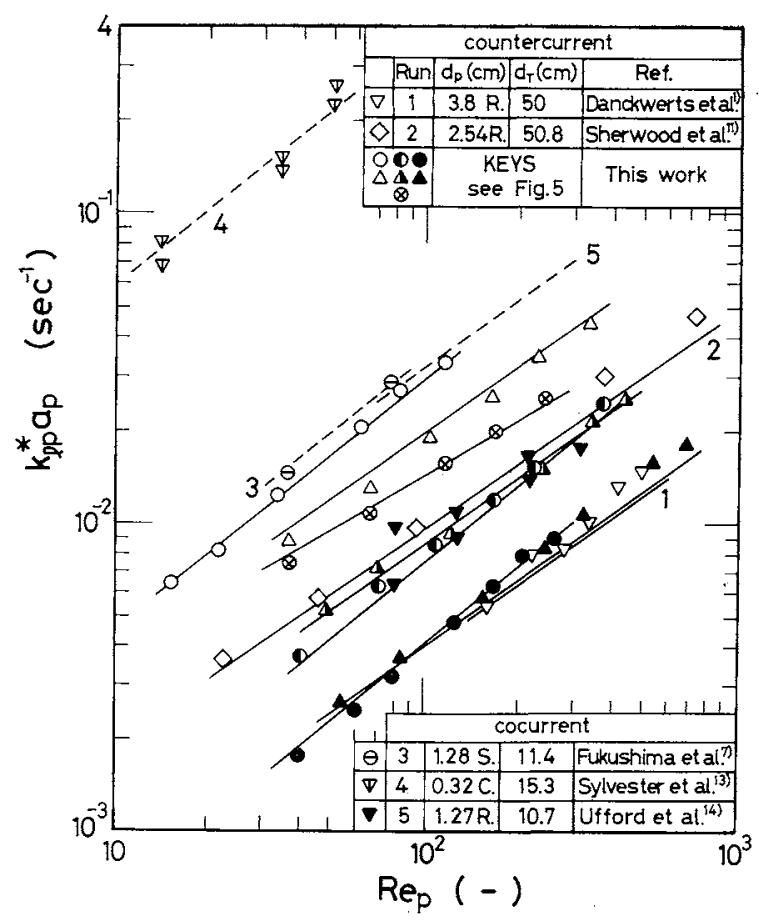

Fig. 8 Dependence of liquid-phase volumetric mass-transfer coefficient on liquid Reynolds number in packed columns

$$
S h_{t}=k_{l t}^{*} d_{p} / D_{A}=7.1 R e_{t}^{1 / 3} S c^{1 / 2}
$$

The prediction agrees with the data with an accuracy of $\pm 10 \%$ in Fig. 7. It is very interesting that the data for one string and the two types of seven strings give the same equation of $k_{l t}^{*}$, because this fact indicates that $k_{l t}^{*}$ is independent of $q$. Thus, it suggests that the liquid trickles on the static holdup of liquid at contact points of spheres as on the spheres.

In comparison with the theory given by Pigford ${ }^{10)}$, Eq. (10) is in the same power of $R e_{t}$ and Schmidt number $S c$, but the constant is a half. The data for a short wetted-wall column reported by Vivian and Behrman ${ }^{15\}}$ are in excellent accordance with the constant and powers in Eq. (10).

The dimensionless equation of liquid-phase volumetric mass-transfer coefficient is presented by multiplying Eqs. (7) and (10) as follows:

$$
k_{l t}^{*} a_{t} d_{p}^{2} / D_{A}=34 q^{-1.1} R e_{t}^{\left(1 / 3+0.27 q^{0.25}\right)} S c^{1 / 2}
$$

The solid lines in Fig. 6 are the predictions given by Eq. (11). Substitution of the data on $k_{l t}^{*} a_{t}$ for two strings into Eq. (11) gives $q=4$, but the actual value of $q$ is 2.7. This result obviously indicates that $m$ must be at least 3 for Eq. (11) to apply.

2) Packed column Figure 8 shows the dependence of liquid-phase volumetric mass-transfer coefficient $k_{l p}^{*} a_{p}$ on $R e_{p}$ in packed columns with countercurrent flow. The data on liquid-phase mass-transfer coefficients $k_{i p}^{*}$ are also obtained by dividing $k_{l p}^{*} a_{p}$ by $a_{p}$. The rearranged data on $k_{b p}^{*}$ give the following equation. 


$$
S h_{p}=\frac{k_{l p}^{*} d_{p}}{D_{A}}=\frac{27}{\phi(1-\varepsilon)} \operatorname{Re}_{p}^{1 / 3} S c^{1 / 2}
$$

where $\phi$ is the surface shape factor of packing. This equation is quite similar to Eq. (10). Prediction by Eq. (12) agrees with the data for Rasching rings, spheres and Berl saddles with an accuracy of $\pm 15 \%$, as shown in Fig. 9.

The dimensionless equation of liquid-phase volumetric mass-transfer coefficients is written by multiplying Eqs. (8) and (12) as follows:

$$
\frac{k_{l p}^{*} a_{p} d_{p}^{2}}{D_{A}}=\frac{1.3 \times 10^{2}}{\phi} q^{-1.1} \operatorname{Re}_{p}{ }^{\left(1 / 3+0.27 q^{0.25}\right)} S c^{1 / 2}
$$

For countercurrent irrigation flow, Fig. 8 shows the correlation of the predictions (solid lines) given by Eq. (13) and the data for spheres and Raschig rings in this work, and for Raschig rings in the literature. The predicted values are in good agreement with the data given by Sherwood and Holloway ${ }^{11}$, and by Danckwerts and Gillham ${ }^{11}$.

There are few available data in the literature for cocurrent irrigation flow. The predictions (broken lines) given by Eq. (13) agree well with the previous data $^{7}$ for spheres, and the Sylvester data ${ }^{13)}$ for cylinders, where the geometrical area of the cylinder is $20 \%$ lower than for Raschig rings and the value of $q$ is supposed to be 10 . The values are higher than the Ufford data ${ }^{14)}$ for Raschig rings by a factor of as much as 3. The empirical equations applicable to the Ufford data ${ }^{14}$ have not been represented. It is desirable to make further investigation of $k_{l p}^{*} a_{p}$ in packed columns with cocurrent irrigation flow.

The practical utility of the multi-strings of touching spheres is described in Appendix 2.

\section{Conclusions}

Conclusions drawn from this investigation on multi-strings of touching spheres are:

1. The number of contact points of spheres per sphere is found to be the important part of interfacial area in multi-strings of touching spheres and in irrigated columns packed with spheres.

2. The liquid-phase mass-transfer coefficient is independent of the number of strings. This indicates that the liquid irrigates the static holdup of liquid at the contact points as well as the spheres in multistrings of touching spheres.

3. The experiments with the laboratory column consisting of three strings of ten touching spheres give usable predictions for packed columns with irrigation flow.

\section{Appendix}

\section{Height of $z_{2}$}

The equation governing absorption of gas into a liquid film running down a vertical cylinder is given by Pigford ${ }^{10)}$. A

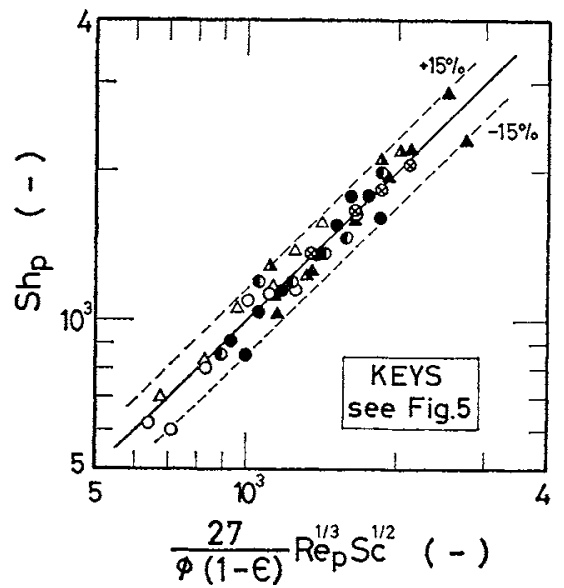

Fig. 9 Correlation of Eq. (12) with the data on packed columns

modified equation of gas absorption on a sphere is presented by Davidson and Cullen ${ }^{3)}$. The film thickness on a sphere at angle $\theta$ is given by

$$
\delta=\left(3 \mu_{l} Q_{t} / \pi \rho_{l} d_{p} g \sin ^{2} \theta\right)^{1 / 3}
$$

The analysis of pictures taken by a Nikon camera gave the following items of information. Below a liquid rate of $13 \mathrm{~cm}^{3}$ / $\mathrm{sec}$, the liquid film was found to be free from ripples in a string of two touching spheres, $1.5 \mathrm{in}$. in diameter. The interface on a sphere, applicable to this equation, was in the range of angle from $0.23 \pi$ to $0.77 \pi$. The length of a sphere equivalent to that of a wetted-wall column is obtained as

$$
l=\frac{d_{p}}{2} \int_{0.23 \pi}^{0.77 \pi} \sin ^{5 / 3} \theta d \theta
$$

The length of the sphere at the top and bottom is given as

$$
l^{\prime}=\frac{d_{p}}{2} \int_{0.16 \pi}^{0.77 \pi} \sin ^{5 / 3} \theta d \theta
$$

The shadow space $\mathbf{P}_{1} \mathbf{P}_{0} \mathbf{P}_{2} \mathbf{P}_{3}$, which is held between the string of touching spheres and the circle, $d_{j}$ in diameter, contacting at $\mathbf{P}_{1}$ and $\mathbf{P}_{2}$ as shown in Fig. A-1, was only $2.5 \%$ lower than the static liquid holdup at contact points between touching spheres. The static liquid holdup for 1 and $1.5 \mathrm{in.} \mathrm{touching} \mathrm{spheres} \mathrm{were}$ 0.14 and $0.22 \mathrm{~cm}^{3}$, respectively. Furthermore, the values of $d_{j}$ for these spheres were 0.61 and $0.92 \mathrm{~cm}$. Hence, $d_{j}=0.24 d_{p}$. It is thus reasonable to predict that the liquid is running down on the arc $\mathbf{P}_{1} \mathbf{P}_{3} \mathbf{P}_{2}$. The arc length equivalent to wetted-wall column is given in the same fashion by

$$
h=d_{j} \int_{0.23 \pi}^{\pi / 2}\left[\frac{\left(d_{p}+d_{j}\right) \sin (0.23 \pi)-d_{j} \sin \theta}{\left\{\left(d_{p}+d_{j}\right) \sin (0.23 \pi)-d_{j}\right\} \sin \theta}\right]^{4 / 3} \sin ^{5 / 3} \theta d \theta
$$

Supposing this analysis applies to liquid flow accompanied by ripples, the height of $z_{2}$ for a string of $N$ touching spheres is written as follows:

$$
z_{2}=2 l^{\prime}+(N-2) l+(N-1) h
$$

Thus, Eq. (2) is given.

2. Practical utility of multi-strings of touching spheres

We discuss here the practical utility of multi-strings of touching spheres on the basis of this investigation, in the case where the resistance of mass transfer in the liquid side is controlling.

The following items of information are obtained from experiments in laboratory columns having three strings and seven strings in closed configuration.

1) Determination of concentration at interface Assume two different numbers of touching spheres in the strings. The equation is given by Eq. (3): 


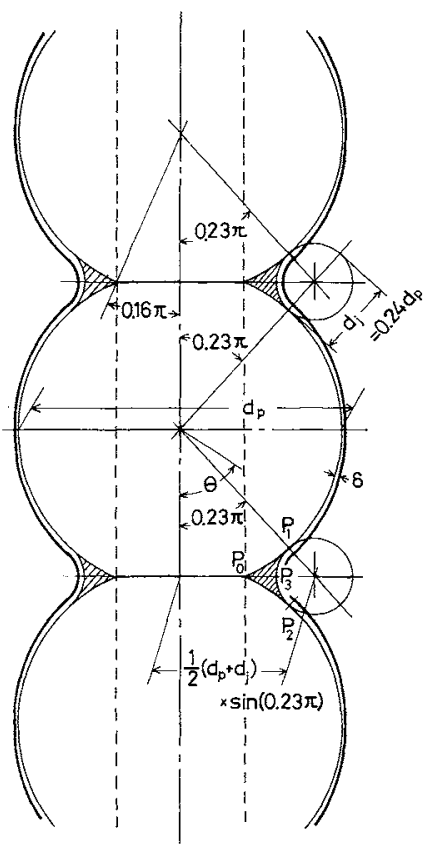

Fig. A-1 Schema of height of $z_{2}$ in a string of touching spheres

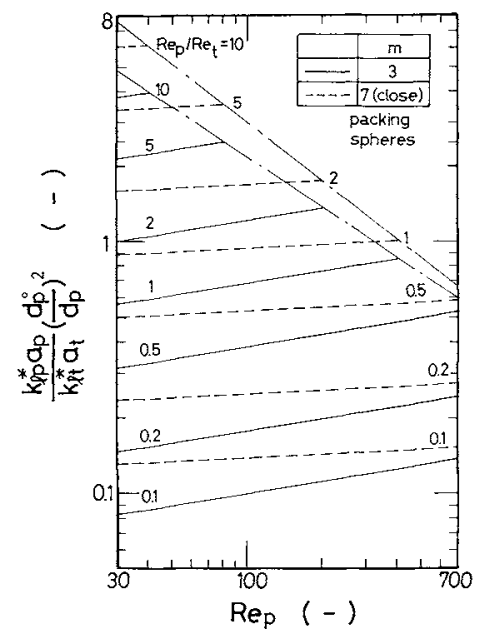

Fig A-2 Correlation of sphere columns with three strings and closed configuration of seven strings of touching spheres

$$
\frac{z_{t 1}}{z_{t 2}}=\frac{\ln \left(1-C_{A z 1} / C_{A i}\right)}{\ln \left(1-C_{A z 2} / C_{A i}\right)}
$$

The concentration of chemical species $A$ in liquid at interface, $C_{A i}$, is thus obtained from the mass transfer rate, even though $m=1$.

2) Predictions of liquid-phase volumetric mass-transfer coefficient in packed column The ratio of the liquid-phase volumetric mass-transfer coefficient in a packed column to that in strings of touching spheres is obtained from Eqs. (11) and (13) as follows:

On the basis of three strings of touching spheres:

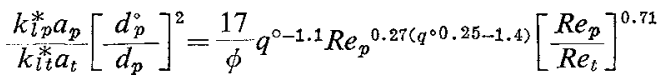

On the basis of seven strings in closed sphere arrangement:

$\frac{k_{l p}^{*} a_{p}}{k_{l t}^{*} a_{t}}\left[\frac{d_{p}^{\circ}}{d_{p}}\right]^{2}=\frac{36}{\phi} q^{0-1.1} \operatorname{Re}_{p}^{0.27\left(q^{\circ 0.25-1.7)}\right.}\left[\frac{R e_{p}}{R e_{t}}\right]^{0.78}$

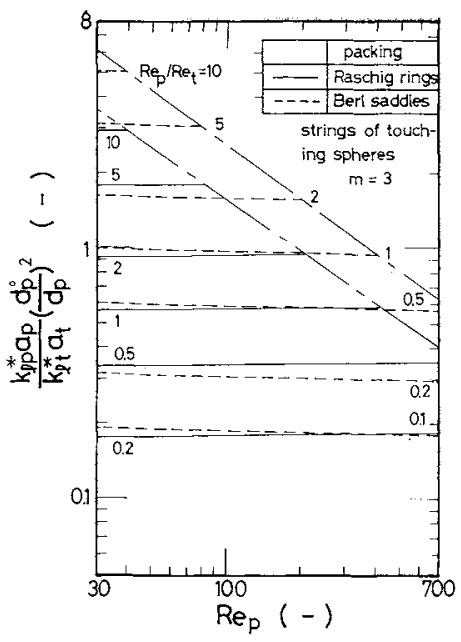

Fig. A-3 Correlation of Raschig ring and Berl saddle columns with three strings and closed configuration of seven strings of touching spheres

where the superscript ${ }^{\circ}$ indicates the values in packed columns. Charts which express Eqs. (A-7) and (A-8), are shown in Figs. A-2 and A-3, respectively. These can be used to predict the mass transfer rate in columns packed with spheres, Berl saddles and Raschig rings from experiments with these strings of touching spheres, where the value of $N$ is larger than 10 .

A column of seven strings in open sphere arrangement is also usable. A column of three strings of touching spheres is the simplest miniplant for this purpose.

\section{Acknowledgment}

The authors express special appreciation to Mr. T. Bunko, Mr. N. Hosokawa, Mr. T. Sakamoto, Mr. Y. Takamura and Mr. E. Ueyama for their useful experimental assistance.

\section{Nomenclature}

$a_{p} \quad=$ interfacial area per column volume $\quad\left[\mathrm{cm}^{-1}\right]$

$a_{t} \quad=$ interfacial area per volume of strings

$\begin{array}{ll} & \text { of touching spheres } \\ C_{A} & =\text { concentration of chemical species } \mathrm{A} \text { in }\end{array}$

$D_{A} \quad=\quad \begin{aligned} & \text { diffusivity of chemical species } \mathrm{A} \text { in } \\ & \text { S }\end{aligned}$

$\left[\mathrm{cm}^{-1}\right]$

solution $\left[\mathrm{cm}^{2} / \mathrm{sec}\right]$

[g-mole/l]

$\begin{array}{lll}d_{j} & =\text { diameter of circle } & {[\mathrm{cm}]}\end{array}$

$d_{p} \quad=$ diameter of touching spheres or packing diameter

$d_{s} \quad=$ equivalent diameter of packing based on sphere

[cm]

$\begin{array}{lll}d_{T} & =\text { diameter of packed column } & {[\mathrm{cm}]}\end{array}$

$G \quad=$ superficial mass velocity $\quad\left[\mathrm{g} / \mathrm{cm}^{2} \cdot \mathrm{sec}\right]$

$g \quad=$ acceleration due to gravity $\quad\left[\mathrm{cm} / \mathrm{sec}^{2}\right]$

$h \quad=$ length at contact point, equivalent to wetted-wall column [cm]

$K, k=$ constant in Eq. (4) [-]

$k^{*} \quad=$ mass-transfer coefficient without chemical reaction

$[\mathrm{cm} / \mathrm{sec}]$

$l=$ length of a sphere equivalent to wettedwall column

[cm]

$l^{\prime} \quad=$ length of sphere at top or bottom of string, equivalent to wetted-wall column $\quad[\mathrm{cm}]$

$m \quad=$ number of strings $\quad[-]$

$N \quad=$ number of touching spheres per string $\quad[-]$

$Q \quad=$ volumetric flow rate $\quad\left[\mathrm{cm}^{3} / \mathrm{sec}\right]$

$q .=$ mean number of contact points of 


$\begin{array}{lll} & \text { touching spheres per sphere } & {[-]} \\ q_{c} & =\text { number of contact points } & \\ q_{\text {max }} & = & \text { maximum number of contact points } \\ R e_{p} & = & \text { liquid Reynolds number of packed } \\ & \text { column, } d_{s} G_{l} / \mu_{l} \\ R e_{t} & = & \text { liquid Reynolds number of } m \text { strings } \\ & \text { of touching spheres based on equator of } \\ & \text { spheres, } 4 \rho_{l} Q_{l} / m \pi d_{p} \mu_{l} \\ = & \text { geometrical surfaces of packing } \\ S & =\text { Schmidt number in liquid phase, } \mu_{l} / \rho_{l} D_{A}\end{array}$

[-] $p \quad=$ packed column

[-] $t \quad=$ strings of touching spheres

\section{Literature Cited}

1) Danckwerts, P. V. and A. J. Gillham: Trans. Inst. Chem. Engrs. (London), 44, T42 (1966).

2) Danckwerts, P. V. and S. F. Rizvi: ibid., 49, 124 (1971).

3) Davidson, J. F. and E. J. Cullen: ibid., 35, 51 (1957).

4) Davidson, J. F., E. J. Cullen, D. Hanson and D. Roberts, ibid., 37, 122 (1959).

5) de Waal, C. J. A. and W. J. Beek: Chem. Eng. Sci., 22, 585 (1967).

6) Fukushima, S. and K. Kusaka: J. Chem. Eng. Japan, 10, 461 (1977).

7) Fukushima, S. and K. Kusaka: ibid., 10, 468 (1977).

8) Jhaveri, A. S. and M. M. Sharma: Chem. Eng. Sci., 23, 66 (1968).

9) Lynn, S., J. R. Straatemeier and H. Kramers: ibid., 4, 63 (1955).

10) Pigford, R. L.: Ph. D. Thesis, Chem. Eng., Univ. of Illinois (1941).

11) Sherwood, T. K. and F. A. Holloway: Trans. AIChE., 36, 21 (1951).

12) Stephens, E. J. and G. A. Morris: Chem. Eng. Progr., 47, 232 (1951).

13) Sylvester, N. D. and P. Pitayagutsarn: Ind. Eng. Chem., Process Des. Dev., 14, 421 (1975).

14) Ufford, R. C. and J. J. Perona: AIChE J., 19, 1233 (1973).

15) Vivian, J. E. and W. C. Behrman: ibid., 11, 656 (1965).

(Presented at the 5th Autumn Meeting of The Soc. of Chem. Engrs., Japan, at Osaka, October, 1971.) 